\title{
Il paesaggio archeologico del castello di Nucetto (Piemonte, Italia): una possibile conservazione
}

The archaeological landscape of Nucetto's Castle (Piedmont, Italy): a possible conservation

\section{Elisa Tartaglino}

Specialista in Beni Architettonici e del Paesaggio - Politecnico di Torino, Turin, Italy, elisa.tartaglino@outlook.com

\begin{abstract}
Each fortified architecture has its own specificities thanks to which defend the territory, it is usually part of garrison systems and has always been a protagonist of the events of the place where it was built. Through this reading can be interpreted the ruins of the Castle of Nucetto (CN, Italy), which insist on the land of Alta Valle Tanaro -mostly located on the south-east portion of Cuneo's territory and in a little part of Savona's one- as real landmark visible from the historical road axis of the valley. The castle's ruins became part of the consolidated image of the landscape thanks to the union they generated with the context giving rise to a recognizable landscape. The castle is part of a wider defensive system intimately linked to the history of the Marquisate of Ceva. Despite the still uncertain reliability of the documents available, the original structure can be dated at the eleventh century. The presence of bands of hanging arches that run along some elevated of the structure allow to hypothesize and recognize a first foundational nucleus while some traces of frescoes rise to appreciable particularities during the visit. The tower, whose base is in brick, but the top part is curiously in stone, is today the best preserved.

The paper aims to analyze the fortification -made with different materials- to understand the extent of the historical stratifications found in the presence of at least two expansions dating from the thirteenth and fifteenth centuries, up to the nineteenth-century destruction wanted by Napoleon. It is also the author's will to propose some suggestions for a possible conservation, starting with an analysis of the state of today's storage (outcome of very limited consolidation interventions operated twenty years ago) to arrive at its insertion in more valuation circuits wide.
\end{abstract}

Keywords: Castle, ruins, landmark, archaeological landscape.

\section{Introduzione ${ }^{1}$}

L'intera Alta Valle Tanaro è disseminata da una serie di fortificazioni riconducibili a un vero e proprio sistema, ormai allo stato di rovina ma ancora leggibile nel suo insieme, a testimonianza di una struttura storica stratificatasi nel corso dei secoli i cui manufatti architettonici presentavano -allora come oggi- un'articolata trama relazionale. Dalla ricognizione puntuale di tali fortificazioni in occasione di due campagne di cono- scenza condotte in team nel periodo 2017-2018 all'interno di attività di atelier della Scuola di Specializzazione del Politecnico di Torino volte ad accertare la permanenza o meno delle architetture rispetto alle fonti documentarie, sono emersi i ruderi del castello di Nucetto. Localizzate nella frazione Villa, su un'altura a meno di dieci chilometri da Ceva - centro che segna l'inizio della Val Tanaro la quale si snoda fino a 
Ormea, ove convenzionalmente termina- le rovine sono facilmente apprezzabili già dalla S.S.28 che corre lungo il fondovalle e assurgono a landmark.

\subsection{Il castello nei secoli}

La scarsa attendibilità delle fonti finora note non consentono di ricostruire le vicende antecedenti al XIII secolo ma, anche se più strutture sono riconducibili a epoche successive, la fondazione della fortificazione è riconducibile al XI secolo (Odello, 2009).

La prima data certa è quella del 1142, momento in cui il Marchesato di Ceva annette ai propri possedimenti il castrum noxeti e il territorio a esso connesso (Palmero, 1998). Giorgio II il Nano, dopo aver conquistato i sopracitati territori a fine XIII secolo, nel 1295 cedette $^{2}$ le sue proprietà alla città di Asti venendone subito reinfeudato e ottenendo protezione militare. Le entrate monetarie derivanti da tale operazione consentirono pertanto un primo ampliamento della struttura (Viglino Davico, Massara, Novelli, 2010; Odello, 1992).

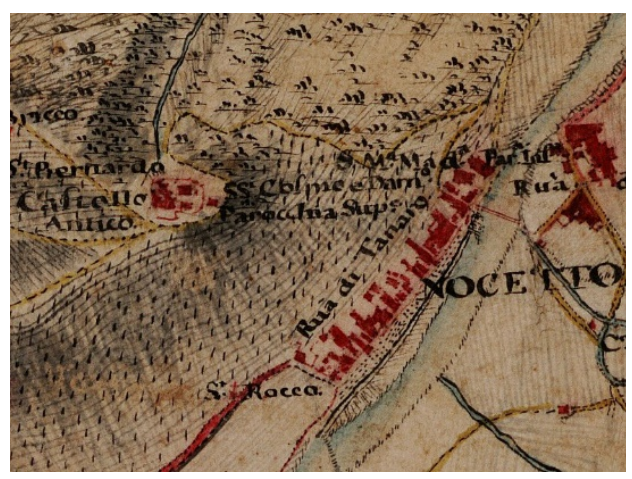

Fig. 1. Particolare dell' abitato di Nucetto e del Castello Antico (ASTo, [XVIII secolo]).

Dopo alcuni avvicendamenti nella giurisdizione feudale, nel 1358 fu Giorgio di Ceva a riacquisire la fortificazione di Nucetto la quale subì ingenti danni a causa delle truppe di Amedeo VIII nel 1414; i marchesi di Ceva dovettero così arrendersi e giurare fedeltà ai Savoia nel 1515 (Odello, 2009). Dopo un primo restauro e un ulteriore ampliamento, il castello fu donato nel 1535 da Giovanni di Ceva a Beatrice di Porto- gallo, duchessa di Savoia. La fortificazione e i possedimenti annessi passarono di mano in mano per via di ripetuti infeudamenti finché nel 1737 divennero proprietà dei Pallavicino (Palmero, 1998; Odello 1992).

Si presume che il castello venne abitato fino a fine XVIII secolo data l'assenza di informazioni in merito a un possibile stato di rovina della fortificazione da parte dell'Intendente Corvesy ${ }^{3}$. Dopo essere stato ridimensionato a semplice caserma a seguito delle campagne napoleoniche e quindi alla originaria mera esigenza difensiva della valle, il manufatto architettonico venne demolito nel 1802 (Odello, 1979).

Il catasto napoleonico del 1804 di Fig. 2 pone in evidenza le chemin du Chateau (leggibile graficamente anche in Fig. 1), e la persistenza delle coltivazioni a vigneto (oggi scomparse); la dicitura Chateau abbandonè evidenzia la condizione di declino della fortificazione e si pone in contrasto con la precedente definizione di Castello Antico (Fig. 1).

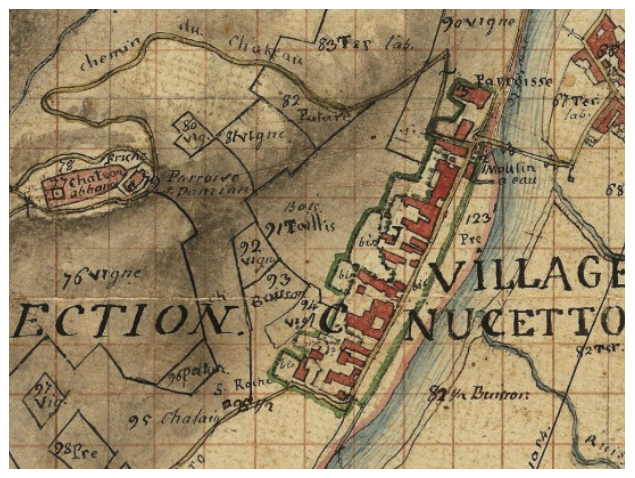

Fig. 2. Stralcio del catasto napoleonico (ASTo, 1804).

\section{Un'analisi del sito e delle rovine}

In prossimità della fortificazione sorgono la canonica e la parrocchiale dei Santi Cosma e Damiano (fondata nel 1594 su una prima cappella afferente al castello), oltre che i resti di un edificio ottocentesco demolito nei primi anni Novanta (Fig. 3). L'area su cui a tutt'oggi insistono fu realizzata in seguito alla parziale demolizione di un bastione perimetrale (XIV secolo), e dell'impostazione di un pianoro realizzato con 
terreno di riporto al fine di poter erigere strutture di servizio come stalle e depositi (Odello, 1979).

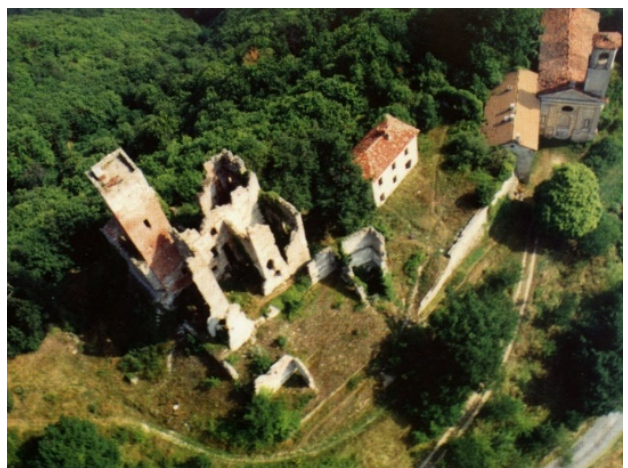

Fig. 3. Foto aerea delle rovine del castello, della palazzina ottocentesca e della parrocchiale nel 1988 (Odello, 2009).

Lo stato di conservazione del castello permette di apprezzare la compresenza di materiali litici e laterizi a riprova di precedenti studi (Viglino Davico, Massara, Novelli, 2010; Odello, 1992, 2009), che individuano due ampliamenti risalenti al XIII e al XV secolo. Le strutture antiche furono difatti riadattate con l'ausilio di laterizi portando la fortezza realizzata a scopo puramente difensivo a diventare l'abitazione del marchese (Odello, 1992). La coesistenza materica si può notare anche nella composizione degli archetti pensili con peducci in pietra -posti in posizione sommitale su alcuni paramenti murari (Fig. 6)che si reputano prova rilevante nel riconoscimento del fabbricato originario rispetto agli interventi successivi.

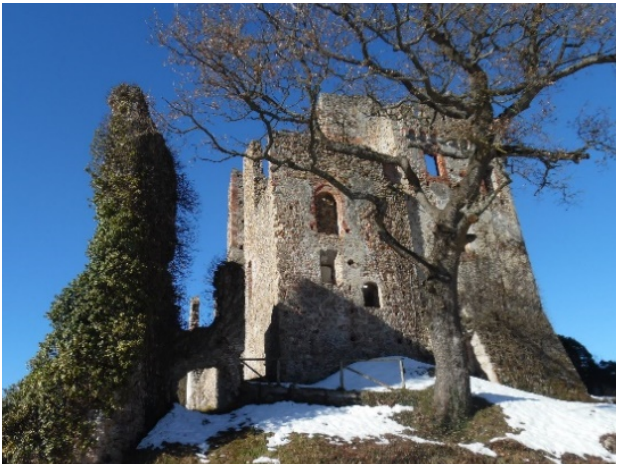

Fig. 4. Vista sud-est delle rovine (Tartaglino, 2019).
La struttura originaria a impianto quadrangolare si trova a nord della attuale conformazione, mantiene una buona vista verso Ceva e alcune aperture sul prospetto est; di queste ne permangono quattro in laterizio -successive alla fondazione dato il confronto con altre morfologicamente simili- due feritoie e un ingresso, mentre altre risultano tamponate.

Gli archetti pensili sulla sommità dell'elevato della scarpa continuano in una fascia con motivo a dentelli seguendo le murature ascrivibili al nucleo di fondazione e collegando al contempo parte del prospetto est (Fig. 4), con il muro di cinta ovest (Fig. 5). Proseguendo intorno alla struttura, gli archetti assumono carattere materico misto e sembrano voler riproporre la decorazione antica (Fig. 7), sull'elevato del prospetto sud.

Risulta inoltre rilevante notare come la realizzazione dei cantonali del lato sud-est del nucleo originario vada a interrompere la fascia di archetti in laterizio, posteriori a quelli litici; tale intervento li riconduce così a un'ulteriore fase costruttiva successiva all'azione sul cantonale (Vallero, 2018).

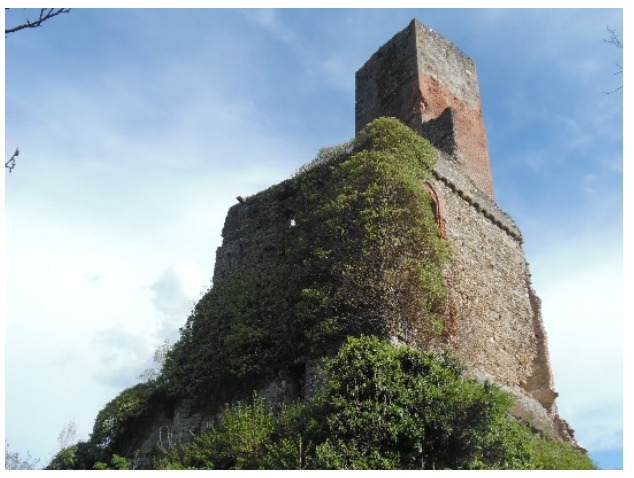

Fig. 5. Vista del prospetto nord-ovest del nucleo antico (Tartaglino, 2019).

Del primo fabbricato sono ancora leggibili gli ambienti interni grazie ad alcune tracce di solai che rinviano a quattro livelli diversi con aperture simili a quelle analizzate in precedenza e a un camino che serviva l'ultimo piano. Altri varchi alcuni coevi alla muratura, altri successivi- e una canna fumaria sono leggibili sull'elevato di 
chiusura dell'antico nucleo che si innesta sulla muratura occidentale in aderenza alla torre.

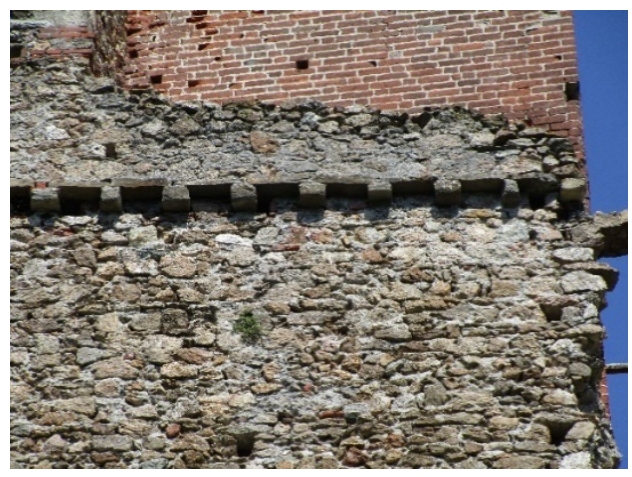

Fig. 6. Gli archetti pensili litici sul prospetto ovest (Tartaglino, 2019).

La torre presenta pianta quadrata con lati di 5,00 $\mathrm{m}$ e, seppur appartenente al primo nucleo della fortificazione, è stata eretta in due momenti distinti. Inizialmente realizzata in pietra e rivestita in laterizio fino quasi alla sua sommità, la parte terminale è stata successivamente ricostruita -a esclusione della merlatura, a tutt'oggi mancantein pietra in appoggio sugli elevati soggiacenti (Fig. 5). Le fondamenta sono costituite da un basamento costituito dal livellamento di rocce preesistenti (Fig. 9), forse rovine di una torre anteriore, su cui poggia un corso di blocchi squadrati seguito da laterizi (Odello, 1992). Sono presenti più accessi e si apprezzano ancora alcune pareti intonacate oltre che i segni di una copertura sul lato nord.

In occasione del primo ampliamento del XIII secolo si tenne conto dell'orientamento a favore di una migliore esposizione dei locali (Odello, 1992): se ne ha conferma con la costruzione di due manufatti che vanno a formare una corte quadrangolare dalle dimensioni contenute e allo stesso punto definiscono il prospetto sud odierno.

Nel primo corpo aggiunto venne realizzata una cucina-destinazione testimoniata dalla presenza di un camino al piano terra e da una cisternamentre alcune aperture al piano superiore suggeriscono fini abitativi. Il secondo manufatto va a interrompere la fascia decorativa di archetti pensili con una modanatura ancora leggibile e la presenza di tracce di una volta a padiglione suggerisce il completamento dell'ambiente.

L'insufficienza dei manufatti pervenuti non permette invece di comprendere che funzioni furono assegnate ai locali derivanti dall'ampliamento del XV secolo anche a causa della loro localizzazione su un pendio piuttosto scosceso, prospiciente all'intervento del XIII.

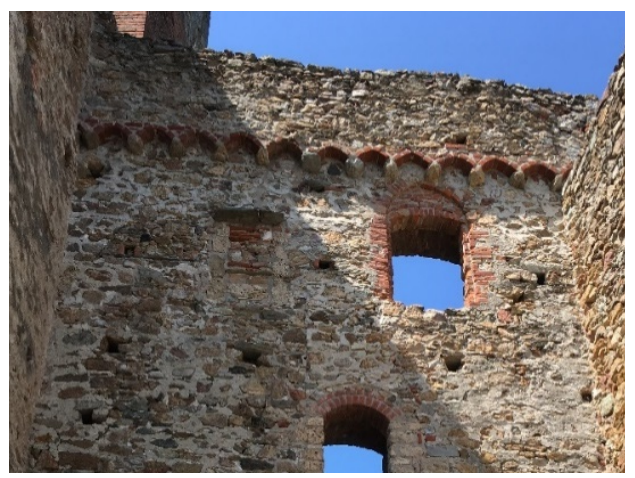

Fig. 7. Gli archetti pensili in laterizio con peducci litici (Tartaglino, 2019).

\subsection{Gli interventi}

Le prime notizie di interventi risalgono rispettivamente al 1836 e al 1936 e sono legate alle strade di accesso alla frazione Villa che necessariamente passano dal castello e dalla parrocchiale (Odello, 2009).

Dal 1946 la fortificazione è tutelata con vincolo monumentale ma il primo progetto di restauro commissionato dall'amministrazione comunale risale al periodo 1999-2000. Obiettivo principale è stato il consolidamento delle strutture murarie sopravvissute operato, secondo la relazione illustrativa, con il ripristino delle parti sommitali degli elevati (con il reimpiego dei materiali collassati rimasti in loco), e delle superfici di sacrificio. Scendendo maggiormente in dettaglio, sono stati eseguiti discutibili cordoli in cemento armato all'interno dei paramenti antichi in pietra in corrispondenza dei perimetri sommitali e ove la sezione muraria era ridotta col fine di colmare le fessurazioni; elementi tipici come aperture e camini sono stati inoltre ripristinati. Una breve campagna di scavi archeologici condotta parallelamente ai lavori, ha consentito di rinvenire il 
piano inferiore del fabbricato antico e parte di una scala, poi consolidati.

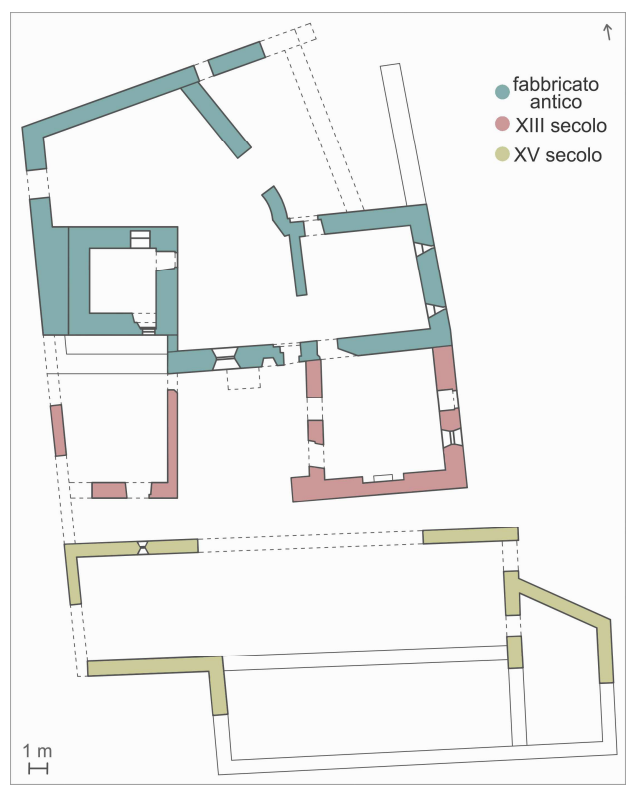

Fig. 8. Rielaborazione grafica della planimetria con indicazione delle principali fasi costruttive (Tartaglino, 2019).

Alle opere finora descritte, si aggiunse la rimozione della vegetazione infestante, la messa in sicurezza dei percorsi (Viglino Davico, Massara, Novelli, 2010), e l'installazione di un impianto dedicato all'illuminazione notturna che permette la percezione delle rovine dalla statale di fondovalle (Odello, 1992).

\subsection{Lo stato attuale}

L'importante interrelazione visiva delle rovine con il paesaggio circostante già osservata dal Casalis nel 1843: “dell'antichissimo castello [...] più non si vedono che la torre, e poche muraglie: bellissima è la positura ove sorgeva: di là si scopre tuta la valle sino al dissopra di Bagnasco" (Casalis, 1972-1977), perdura con forza ancora oggi e rende il sito fortificato un punto di notevole belvedere.

Le opere sul manufatto svolte ormai vent'anni orsono, forse per via della loro prepotenza in certe parti, consentono comunque alla struttura di presentarsi in un discreto stato di conserva- zione dato il raffronto con la documentazione fotografica depositata presso l'amministrazione comunale. Nonostante la non semplice leggibilità delle parti ancora intonacate tra cui quella con il motivo a spirale su un elevato dell'ampliamento sud e quella di colore nero sull'alzato a est facente riferimento allo stemma del Marchesato di Ceva, non sussistono situazioni critiche all'interno del sito fortificato.

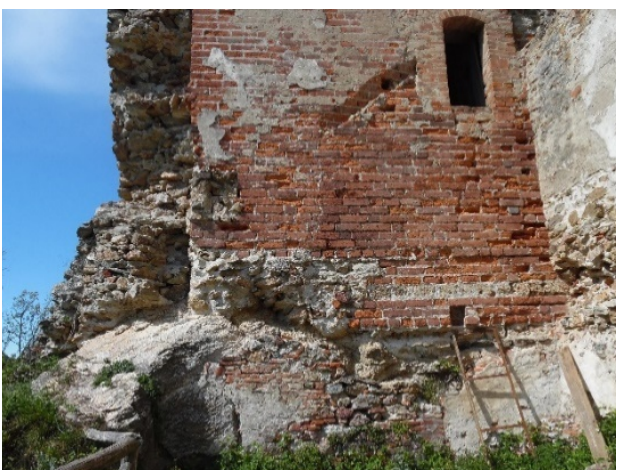

Fig. 9. La parte basamentale della torre (Tartaglino, 2019).

Ugolini asserisce che: "[...] considerato che la vita di un monumento è strettamente connessa all'ambiente in cui si trova, è evidente che la sua conservazione viene influenzata in maniera importante dalla presenza della vegetazione spontanea che lo colonizza o dalle strutture vegetali progettate che lo circondano" (Ugolini, Matteini, 2016, p. 462). Pertanto, anche in tale sito, la vegetazione ruderale possiede un ruolo importante da cui deriva un valore di memoria e un conseguente valore d'uso (Romeo, 2013), da tenere presente sia in fase di conservazione che di valorizzazione.

La materia vegetale che solitamente si insedia in siti archeologici è tra le cause principali di degrado e, a causa degli scarsi fondi da deputare alla manutenzione, spesso ne consegue una eradicazione sconsiderata che non tiene conto né dell'immagine romantica creatasi nel tempo, né della capacità di contenimento e sostegno offerta dall'apparato radicale della vegetazione andando così a peggiorare la stabilità statica delle rovine.

Presso il sito del castello di Nucetto è stato possibile apprezzare un coscienzioso intervento di 
rimozione della materia infestante grazie al confronto della situazione attuale con la documentazione fotografica degli ultimi tre anni.

\section{Proposte di intervento}

In un territorio disseminato di rovine fortificate come quello preso in esame in occasione di tali studi, ci si è resi conto che sono da tenere in forte considerazione interventi consapevoli volti a una integrazione del rudere con la vegetazione al fine di non snaturare la natura intrinseca del luogo.

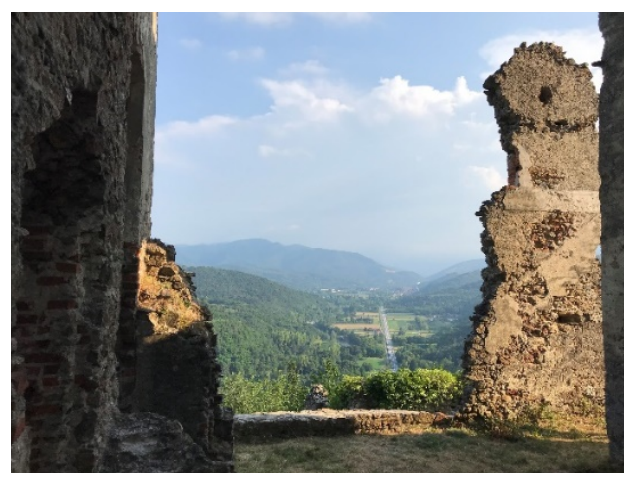

Fig. 10. Veduta verso sud con chiara percezione della S.S. 28 (Tartaglino, 2019).

Da un raffronto puntuale con casi studio europei di architetture fortificate allo stato di rudere è emerso che, per il sito di Nucetto, sarebbero auspicabili interventi come quelli operati nel caso di Rocca San Silvestro (LI), e per il castello dei Doria a Dolceacqua (IM). Il primo ha il merito di aver avvicinato la comunità non solo scientifica tramite una sapiente campagna divulgativa che procedeva di pari passo con le attività di cantiere; il secondo ha riguardato in special modo la progettazione di una nuova e maggiore accessibilità agli ambienti con strutture poco invasive.

Dopo una fondamentale fase di conoscenza del manufatto è pertanto necessario fissare i limiti degli interventi da svolgere ed è altrettanto rilevante documentarne lo stato di avanzamento al fine di poter essere in grado di comprendere in un secondo momento le scelte operate ed essere così di supporto per ulteriori progetti. Resta chiara l'importanza dei cantieri legati alla manu- tenzione, sia ordinaria che straordinaria, per non rischiare di vanificare ciò che è stato fatto durante il cantiere di restauro e sarebbe inoltre auspicabile portare avanti opere di educazione sia nei confronti delle istituzioni, che delle comunità che usufruiscono del patrimonio oggetto di valorizzazione.

\section{Conclusioni}

Le rovine di Nucetto meritano alcune riflessioni di più ampio respiro nonostante insistano su un territorio poco conosciuto e dal valore relativo se raffrontate con patrimoni maggiori (Rudiero, 2015).

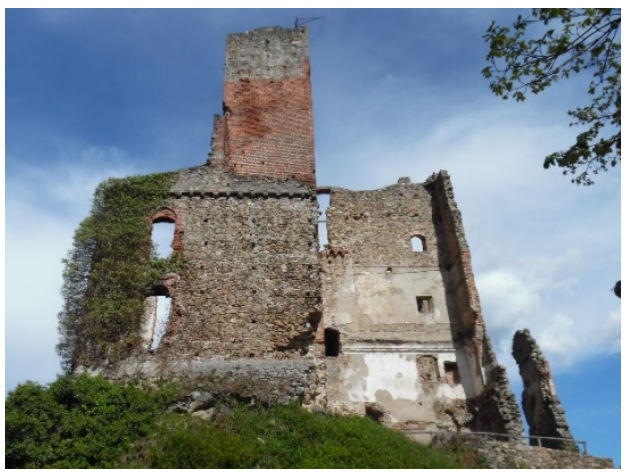

Fig. 11. Prospetto ovest (Tartaglino, 2019).

Fiorani (Fiorani, 2014) prende nota della variazione di tendenza nelle ultime carte del restauro -Convenzione per la salvaguardia del patrimonio culturale immateriale dell'Unesco, 2003; Convenzione di Faro, 2005- per quanto riguarda una maggiore attribuzione di valori dal patrimonio tangibile a quello intangibile dando pertanto maggior rilievo all'aspetto percettivo. L'eredità culturale definita dalla Convenzione di Faro sembra però entrare in conflitto con il dibattito scientifico attuale ove ci si interroga inoltre sul perché restaurare e il per chi (Pane, 2017). Da qui deriva l'importanza delle comunità locali che abitano i territori su cui insiste il patrimonio e il loro senso di appartenenza può diventare l'ago della bilancia per una conservazione riuscita; ciò può accadere però solo nel momento in cui gli vengono forniti strumenti congrui per il riconoscimento e l'accettazione del valore del bene (Rudiero, 2015). Un'opera in tale direzione po- 
trebbe essere il collocamento di cartellonistica dedicata con QR code che permetta al turista di fruire di contenuti divulgativi come testi o brevi video riguardanti, ad esempio, le tecniche costruttive seguite nel castello piuttosto che la messa in evidenza dei diversi ampliamenti architettonici operati nel corso dei secoli.

Interventi volti a una maggiore accessibilità del patrimonio fortificato allo stato di rudere -tema trattato ampiamente negli ultimi anni (Picone, 2014)- rischiano invece di mortificare il sito di Nucetto per il quale sarebbe sufficiente un leggero intervento di messa in evidenza dei tratti percorribili.

\section{Note}

${ }^{1}$ La stesura di questo contributo parte dalle ricerche svolte per la tesi di specializzazione con relatore il Prof. Arch. Emanuele Romeo, discussa presso il Politecnico di Torino nel maggio 2019.

${ }^{2}$ Tra il XIV ed il XV secolo furono recuperati i diritti feudali qui alienati (Palmero, 1998).

${ }^{3}$ Pertanto successivo alla stesura del suo testo (Comino, 2003).

\section{Bibliography}

ASTo, Sezione Corte, Carte topografiche e disegni, Carte topografiche per A e per B, Tanaro, mazzo 1, foglio 2.

ASTo, Catasti, Sezioni Riunite, Catasto francese, Allegato A, Mazzo 72. Mappe del catasto francese, Circondario di Mondovì, Mandamento di Bagnasco, Nucetto.

Carrara, S.; Odello, G. (2014). "Castelli e fortificazioni sul territorio dell'antico Marchesato di Ceva: censimento delle strutture e prime considerazioni”, in Comba, R.; Coccoluto, G., Ceva e il suo Marchesato fra trecento e quattrocento, Bollettino della Società per gli studi storici, archeologici e artistici della provincia di Cuneo, 150, pp. $37-56$.

Casalis, G. (1972-7). Dizionario geografico, storico, statistico, commerciale degli stati di S.M. il re di Sardegna, Forni Editore, Bologna, vol. XII, pp. 7-11.

Comba, R. (2012). "Ceva e il suo Marchesato. Nascita e sviluppi di una signoria territoriale”, Bollettino della Società per gli studi storici, archeologici e artistici della provincia di Cuneo, 146.

Comino, G. (2003). Descrizione della provincia di Mondovì: relazione dell'intendente Corvesy, Centro studi monregalesi, Mondovì.

Fiorani, D. (2014). "Materiale/Immateriale. Frontiere del restauro", Materiali e strutture. Problemi di conservazione, 5-6, pp. 9-23.

Gazzola, P. (1965). "Un patrimonio storico da salvare: i castelli”, Castellum, 1, pp. 7-16.

Marino, B.G. (2019). Across the Stones. Immagini, paesaggio e memoria. La conoscenza interdisciplinare della Fortezza del Girifalco, Editori Paparo, Roma.

Odello, G. (1979). Vicende storiche ed altre notizie di Nuceto e dintorni, Carrù.

Odello, G. (1992). La communità del luogo di Nuceto durante il secolo XVIII: cronaca dagli ordinati comunali, Stilgraf, Vicoforte.

Odello, G. (2009). I De Nuceto, i Nuceto di Cavallerleone, i marchesi Ceva di Nucetto, Stilgraf, Vicoforte, p. 149.

Palmero, B. (1998). Schede storico-territoriali dei comuni del Piemonte, Comune di Nucetto.

Pane, A. (2017). "Per un'etica del restauro", in Fiorani, D., coord., RICerca/REStauro, Edizioni Quasar, Roma, pp. $120-133$.

Picone, R. (2014). Pompei accessibile: per una fruizione ampliata del sito archeologico, L'Erma di Bretschneider, Roma.

Romeo, E. (2013). "Dinamiche, disincanti, dissolvenze nelle rovine del paesaggio", in Regione Autonoma Valle d'Aosta, AVER - Ancien vestiges en ruine, Sarre, AVI Presse, pp. 341-350.

Romeo, E.; Morezzi, E. (2016). Che almeno ne resti il ricordo, Ermes, Ariccia. 
Rudiero, R. (2015). "La conservazione «in progress» di un insediamento alpino: il caso di Santa Giulitta", in Devoti, C.; Naretto, M.; Volpiano, M., Studi e ricerche per il sistema territoriale alpino occidentale. Gubbio, ANCSA, pp. 485-497.

Tartaglino, E. (2019). Paesaggio archeologico e architetture fortificate. Dal dibattito internazionale alle proposte di intervento sul Castello di Nucetto, Relatore Prof. Arch. Romeo E., Tesi di specializzazione, Politecnico di Torino, Torino.

Ugolini, A. (2010). Ricomporre la rovina, Alinea Ed., Firenze.

Ugolini, A.; Matteini, T. (2016). "Trasformando lo sguardo. Il ruolo della vegetazione nella conservazione dei manufatti allo stato di rudere", in Biscontin, G.; Driussi, G., Eresia ed ortodossia nel restauro. Progetti e realizzazioni. Atti del XXXII Convegno di studi internazionale Scienza e Beni Culturali (Bressanone, 28 giugno- $1^{\circ}$ luglio 2016), Arcadia Ricerche, Venezia.

Vallero, S. (2018). Le fortificazioni della Valle Tanaro: analisi di un sistema tardo medievale, Tesi di specializzazione, Politecnico di Torino, Torino.

Viglino Davico, M.; Massara, G.G.; Novelli, F. (2010). Atlante castellano: strutture fortificate della provincia di Cuneo, Celid, Torino. 